\title{
Deoxynivalenol Detoxification in Transgenic Wheat Confers Resistance to Fusarium Head Blight and Crown Rot Diseases
}

\author{
Giulia Mandalà, Silvio Tundo, ${ }^{+}$Sara Francesconi, Federica Gevi, Lello Zolla, Carla Ceoloni, ${ }^{\dagger}$ and \\ Renato D'Ovidio
}

Department of Agricultural and Forest Sciences (DAFNE), University of Tuscia, 01100 Viterbo, Italy

Accepted 10 November 2018.

Fusarium diseases, including Fusarium head blight (FHB) and Fusarium crown rot (FCR), reduce crop yield and grain quality and are major agricultural problems worldwide. These diseases also affect food safety through fungal production of hazardous mycotoxins. Among these, deoxynivalenol (DON) acts as a virulence factor during pathogenesis on wheat. The principal mechanism underlying plant tolerance to DON is glycosylation by specific uridine diphosphate-dependent glucosyltransferases (UGTs), through which DON-3- $\beta$-D-glucoside (D3G) is produced. In this work, we tested whether DON detoxification by UGT could confer to wheat a broad-spectrum resistance against Fusarium graminearum and $\boldsymbol{F}$. culmorum. These widespread Fusarium species affect different plant organs and developmental stages in the course of FHB and FCR. To assess DON-detoxification potential, we produced transgenic durum wheat plants constitutively expressing the barley HvUGT13248 and bread wheat plants expressing the same transgene in flower tissues. When challenged with $\boldsymbol{F}$. graminearum, FHB symptoms were reduced in both types of transgenic plants, particularly during early to mid-infection stages of the infection progress. The transgenic durum wheat displayed much greater DON-to-D3G conversion ability and a considerable decrease of total DON+D3G content in flour extracts. The transgenic bread wheat exhibited a UGT dose-dependent efficacy of DON detoxification. In addition, we showed, for the first time, that DON detoxification limits FCR caused by $F$. culmorum. FCR symptoms were reduced throughout the experiment by nearly $50 \%$ in seedlings of transgenic plants constitutively expressing HvUGT13248. Our results demonstrate that limiting the effect of the virulence factor DON via in planta glycosylation restrains FHB and FCR

Current address for Silvio Tundo: Department of Land, Environment, Agriculture and Forestry (TeSAF), Research Group in Plant Pathology, University of Padova, 35020 Legnaro (Padova), Italy.

${ }^{\dagger}$ Corresponding authors: S. Tundo; silvio.tundo@unipd.it and C. Ceoloni; ceoloni@unitus.it

Funding: This work was funded through financial support from MIUR (Italian Ministry of Education, University and Research), grant "PRIN (Progetti di Ricerca scientifica di rilevante Interesse Nazionale) 2010-11", as well as from Lazio region, FILAS project "MIGLIORA". The research was also partially supported by MIUR in the context of the initiative "Departments of excellence" (law 232/216). G. Mandalà was supported by a Ph.D. fellowship from the University of Tuscia ('Dottorato XXX ciclo') and F. Gevi by a post-doctoral research grant from CIB (Italian Interuniversity Consortium for Biotechnologies).

(c) 2019 The American Phytopathological Society development. Therefore, ability for DON detoxification can be a trait of interest for wheat breeding targeting FHB and FCR resistance.

Fusarium graminearum and F. culmorum are the main causal agents worldwide of Fusarium head blight (FHB) and Fusarium crown rot (FCR) of small grain cereals including wheat. Both diseases cause significant reductions in wheat yield and quality and major economic loss. Seeds from diseased plants not only have lower processing value but are also contaminated by mycotoxins that can affect animal and human health (Pestka 2010). The mycotoxin risk in the food chain is considered particularly threatening in durum wheat production, as durum wheat-derived products are used almost exclusively for human consumption. Trichothecenes are among the most important Fusarium mycotoxins. In wheat, predominant trichothecenes are deoxynivalenol (DON) and nivalenol (NIV), both acting as virulence factors (Maier et al. 2006).

FHB, mainly caused by $F$. graminearum, produces typical symptoms on the spike, such as necrosis of the infected spikelets and spike bleaching due to vessel occlusion. The rachis node seems to have a central role in wheat resistance (Jansen et al. 2005; Miller et al. 2004), as it hinders fungal advancement toward the vascular bundles. TRI5, the first gene of the trichothecene biosynthetic pathway, is highly induced at the rachis node and so is DON production (Boenisch and Schäfer 2011; Ilgen et al. 2009). At the rachis node, cell-wall thickening was developed by a susceptible wheat cultivar infected with a trichothecene-deficient mutant of $F$. graminearum, and this prevented hyphal penetration into the rachis (Jansen et al. 2005). DON is thus considered essential for fungal spread along the spike, although it is not essential for the initial establishment of the infection (Bai et al. 2001).

FCR is another important wheat disease that causes seedling death or tiller abortion depending on the time of infection. FCR leads to a reduction in plant vigor, number of heads, and grain yield and mycotoxin contamination of wheat stubble and heads (Scherm et al. 2013). Among FCR causal agents, F. culmorum is the most aggressive seedling pathogen (Dyer et al. 2009). Although DON does not seem to be a key factor for initial seedling infection, the reduction in virulence of DON-mutant Fusarium strains (Mudge et al. 2006; Powell et al. 2017; Scherm et al. 2011) suggests that DON plays a role in fungal progression through the stem.

Toxicity of DON is mainly due to its inhibitory effect on protein synthesis; the epoxide group on C12/C13 and the hydroxyl group on C3 (Karlovsky 2011) interfere with the activity 
of peptidyltransferase by binding the 60S subunit of eukaryotic ribosomes (Dellafiora et al. 2018; Payros et al. 2016; Ueno et al. 1973; Wei et al. 1974). Moreover, in wheat plants, high DON concentrations induce production of reactive oxygen species, apoptosis-like processes with chlorotic and necrotic lesions, and root-growth inhibition (Desmond et al. 2008; Masuda et al. 2007). Fungal and plant DON derivatives are found in cereal products (Berthiller et al. 2005). Although less active than DON, these 'masked' DON-derived molecules can release their toxic precursor after hydrolysis. The main fungal derivatives are 3- and 15-acetyl DON (3ADON, 15ADON), while the plant derivative is DON-3- $\beta$-D-glucoside (D3G), produced by glucose conjugation on $\mathrm{C} 3$. DON glycosylation by uridine diphosphate-dependent glucosyltransferases (UGTs) (Bowles et al. 2006) has been identified as the main detoxification strategy adopted by the wheat plant (Berthiller et al. 2005; Kluger et al. 2015). Notably, the ability to detoxify DON by glycosylation has been correlated with Fhbl, a major wheat quantitative trait locus (QTL) for FHB resistance (Lemmens et al. 2005). Among known monocot UGTs that detoxify DON, the barley HvUGT13248 conferred DON resistance when heterologously expressed in DON-sensitive yeast (Schweiger et al. 2010) and in Arabidopsis thaliana (Shin et al. 2012). More importantly, the constitutive expression of HvUGT13248 in transgenic bread wheat conferred FHB resistance toward DON- and NIV-producing $F$. graminearum strains (Li et al. 2015, 2017).

Susceptibility against Fusarium spp. infection seems to be higher in durum wheat than in bread wheat cultivars (Buerstmayr et al. 2009; Stack et al. 2002). This could be explained by the fact that durum wheat, traditionally cultivated in warm and dry Mediterranean conditions, has had limited exposure to relevant disease pressure (Ban et al. 2005). Moreover, durum wheat seems to possess strong susceptibility factors for FHB (Ban and

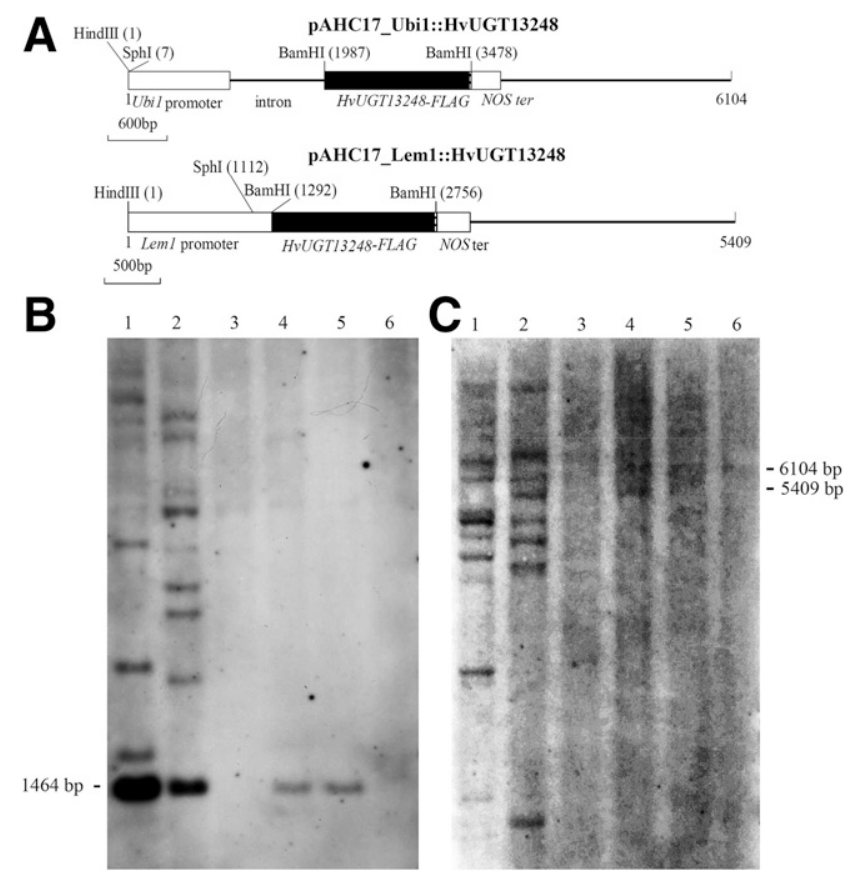

Fig. 1. Southern blot analysis of Ubi-UGT (transgenic lines ST7-47 and ST7-56) and Lem-UGT (transgenic lines ST8-49 and ST8-74) plants. A, The pAHC17_Ubi1::HvUGT13248 and pAHC17_Lem1::HvUGT13248 linearized constructs with main restriction sites. B, Genomic DNA $(10 \mu \mathrm{g})$ of transgenic lines digested with BamHI and $\mathbf{C}, S p h \mathrm{I}$ and probed with a digoxigenin-labeled coding region of $H v U G T 13248-F L A G$. Lanes: 1, ST747; 2, ST7-56; 3, Triticum durum 'Svevo'; 4, ST8-74; 5, ST8-49; and 6, T. aestivum 'Bobwhite'.
Watanabe 2001; Ban et al. 2005), and some of its morphological traits, mainly at the spike level, apparently favor disease development (Lops et al. 1998). The current lack of effective resistance sources is also the result of so-far limited breeding efforts targeting Fusarium resistance in durum wheat compared with bread wheat (Giancaspro et al. 2016; Prat et al. 2017). Thus, to assess the efficacy of DON detoxification for Fusarium resistance in durum wheat, in this work, we produced transgenic durum wheat plants constitutively expressing the barley HvUGT13248 and tested their reaction to FHB and FCR upon infection with $F$. graminearum and $F$. culmorum, respectively.

In previous work, we demonstrated that the transgenic expression in bread wheat spike tissues of the bean PvPGIP2, an inhibitor of $F$. graminearum polygalacturonase activity, provided the same level of FHB resistance exhibited by plants expressing the same gene in all plant tissues (Tundo et al. 2016a). In this work, we also expressed in bread wheat the HvUGT13248 under the control of the Lem 1 promoter, which regulates expression in lemma, palea, rachis, and anthers (Somleva and Blechl 2005; Tundo et al. 2016a), to assess whether DON detoxification in spike tissues could also be sufficient to enhance FHB resistance.

\section{RESULTS}

Production and selection of transgenic durum wheat constitutively expressing $H v U G T 13248$ and transgenic bread wheat expressing $\mathrm{HvUGT13248}$ in floral tissues.

Biolistic transformations of embryogenic calli of durum wheat cultivar Svevo and bread wheat cultivar Bobwhite were performed by cobombardment of the pAHC17_Ubi1:: HvUGT13248 or the pAHC17_Lem1::HvUGT13248, respectively, and the pBI_Ubi1::Bar as selectable marker. The promoters of maize Ubiquitinl and of barley Leml were used for constitutive and floral-specific expression, respectively. The durum wheat bombardment, named ST7, was performed on 1,104 embryogenic durum wheat calli and it produced $13 \mathrm{~T}_{0}$ lines containing the HvUGT13248 transgene (1.18\% transformation efficiency). In the bread wheat experiment, named ST8, of 1,272 bombarded embryogenic calli, four $\mathrm{T}_{0}$ lines presented the HvUGT13248 (0.3\% efficiency). The particularly low transformation efficiency of pAHC17_Lem1-driven constructs was also observed in previous studies (Tundo 2015). Transgenic lines ST7-47 and ST7-56 (hereafter referred to as Ubi-UGT) as well as ST8-49 and ST8-74 (hereafter referred to as Lem-UGT) were selected for further characterization in subsequent generations. None of the analyzed lines exhibited evident phenotypic alterations versus wild-type plants.

\section{Characterization of selected Ubi-UGT and Lem-UGT transgenic lines.}

To confirm transgene integration, genomic DNAs of the selected transgenic lines were analyzed by Southern blot analysis, using about $800 \mathrm{bp}$ of the $H v U G T 13248$ coding region as probe. Cleavage with the BamHI restriction enzyme, which causes the excision of the HvUGT13248 coding region (Fig. 1A), confirmed the presence of the expected hybridization fragment of about $1,400 \mathrm{bp}$ in all transgenic lines (Fig. 1B). In particular, the signal of the ST7-47 line was more intense than that of the ST7-56 line (Fig. 1B), probably reflecting a higher copy number of integrated transgenes. Conversely, both Lem-UGT transgenic lines displayed a similar band intensity (Fig. 1B), which was weaker than that of Ubi-UGT lines, suggesting a lower copy number of integrated coding regions in the former lines. Digestion with the SphI enzyme, which has one 
restriction site within the constructs (Fig. 1A), produced the expected hybridization fragment in all the transgenic lines, corresponding to entire constructs (Fig. 1C) of 6,104 and 5,409 bp for pAHC17_Ubi1::HvUGT13248 and pAHC17_Lem1::HvUGT13248, respectively. In both ST7 lines, additional hybridizing fragments were found (Fig. 1C), indicating the possible occurrence of transgene rearrangement events. Hybridization bands in the ST8 lines were weakly visible (Fig. 1C), reflecting the low copy number of integrations observed with BamHI digestions (Fig. 1B). As expected, in all Southern blot experiments, no hybridization signal was detected in genomic DNA of the untransformed plants.

Reverse transcription-polymerase chain reaction (RT-PCR) of the HvUGT13248 complete coding region was performed to confirm transgene expression. Among durum wheat Ubi-UGT lines (Fig. 2A), ST7-47 produced a more intense band, suggesting a higher transcript level. Concerning Lem-UGT lines (Fig. 2A), HvUGT13248 expression was confirmed in both bread wheat lines, although ST8-49 exhibited a very low level of expression.

Expression in plant tissues of the HvUGT13248 protein was confirmed by Western blot analysis, detecting the FLAG-tag present at the $\mathrm{N}$-terminus of the protein. The signal corresponding to $52 \mathrm{kDa}$, the expected mass for HvUGT13248, was present in both Ubi-UGT lines (Fig. 2B). Two signals of 25 and $20 \mathrm{kDa}$ were also detected, suggesting a partial proteolytic degradation. In Lem-UGT plants, the same analysis revealed the presence of HvUGT13248 protein only in the ST8-74 line (Fig. 2B); the failure of protein detection in the ST8-49 line possibly reflects the corresponding weak transcript level (Fig. 2A). As expected, no signal of HvUGT13248 was detected in the Western blot assay of protein extracted from the untransformed plants.

\section{Constitutive expression of $\mathrm{HvUGT13248}$ \\ in Ubi-UGT durum wheat reduces}

the negative effect of DON on root elongation.

As a DON-inhibiting effect on root elongation was reported (Masuda et al. 2007), an in-vitro root-growth assay was carried out to assess the response to DON of Ubi-UGT durum wheat lines. As shown in Figure $3 \mathrm{~A}$, in the presence of DON, root elongation in both ST7-47 and ST7-56 lines was significantly

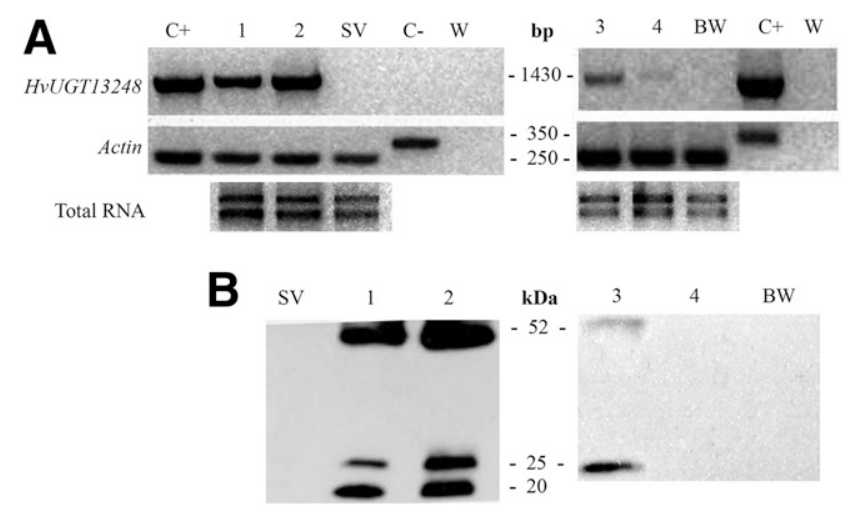

Fig. 2. Expression analyses of Triticum durum 'Svevo' and Ubi-UGT (transgenic lines ST7-47 and ST7-56) plants (leaves) and of T. aestivum 'Bobwhite' and Lem-UGT (transgenic lines ST8-49 and ST8-74) plants (spikelets at anthesis stage). A, Reverse transcription-polymerase chain reaction amplification products of $H v U G T 13248$ complete coding region, of housekeeping Actin gene, and total RNA. B, Western blot detection of the FLAG-tag present at the N-terminus of HvUGT13248 (52 kDa). Lanes: C+, cDNA or gDNA control; 1, ST7-56; 2, ST7-47; SV, T. durum Svevo; Cnull-segregate gDNA control; 3, ST8-74; 4, ST8-49; BW, T. aestivum Bobwhite; and $\mathrm{W}$, water. less inhibited than in the control, although the expression of the barley UGT did not restore normal root growth in the transgenic lines. Compared with the respective mock treatments, root lengths of ST7-47 and ST7-56 lines were reduced by 67 and $39 \%$, respectively, while that of Svevo wild-type plants was reduced by almost $90 \%$ (Fig. 3B).

\section{Resistance to FHB and FCR increases in transgenic Ubi-UGT durum wheat.}

Following spike point-inoculation with $F$. graminearum, symptom progression was slower in both Ubi-UGT lines than in untransformed Svevo plants (Fig. 4A). The maximal reduction of $30 \%$ in symptom severity was between 5 and 9 days postinfection (dpi). During that time period, several transgenic plants showed a temporary block of the infection to the inoculated spikelets. Subsequently, the infection resumed and the difference in symptoms between transgenic and wild-type plants progressively decreased, so that it was no longer significant at $15 \mathrm{dpi}$.

At maturity stage, kernels from infected spikes of the transgenic Ubi-UGT plants were more developed and filled than those of untransformed plants, probably reflecting the longer period elapsed before fungal spreading as compared with the untransformed plants. Thousand kernel weight (TKW, in grams) varied considerably between infected transgenic lines

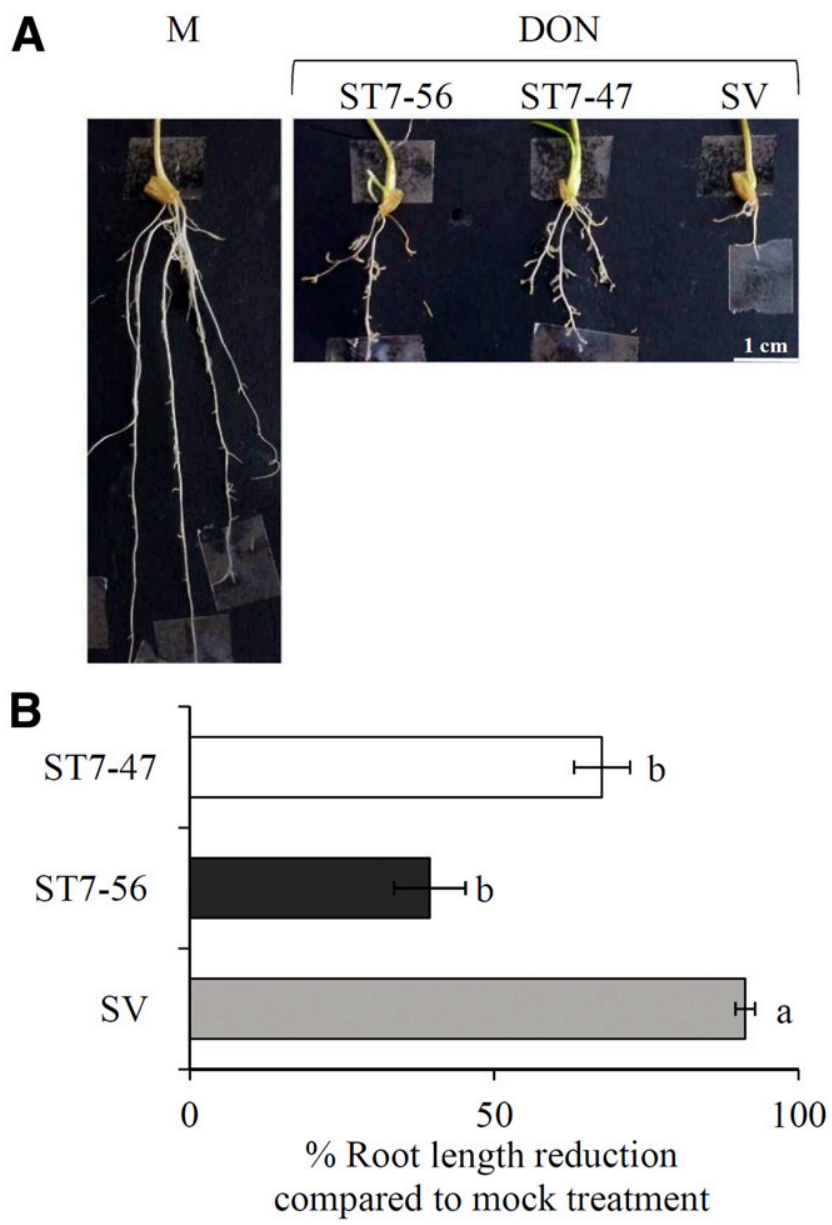

Fig. 3. Root-growth assay on Ubi-UGT (transgenic lines ST7-47 and ST756) and Triticum durum 'Svevo' plants. A, Roots subjected to mock (M) and $10 \mu \mathrm{M}$ deoxynivalenol (DON) treatments. B, Inhibition of root elongation in DON treatment compared with mock. Data represent means \pm standard errors of at least three biological replicates. All data were subjected to analysis of variance. Different letters correspond to significant differences $(P<0.05)$ according to the Tukey test. 
and wild-type Svevo plants; values (mean \pm standard error) were, on average, $10.5 \pm 3.7,9.2 \pm 2$, and $4.2 \pm 2$ for ST7-47, ST7-56, and Svevo, respectively, although differences were not statistically significant. Ultrahigh-performance liquid chromatography (UHPLC) mass spectrometry analyses were performed to quantify DON and D3G contents in flour from mature kernels of Ubi-UGT and untransformed plants (Table 1). Compared with untransformed Svevo, a highly significant reduction in DON+D3G content was detected in both transgenic lines, being about 70 times lower in ST7-47 and over 500 times lower in ST7-56. Irrespective of the difference between the two lines, both exhibited similar D3G/DON ratios, averaging around 20 to $30 \%$. By contrast, the same ratio was $0.3 \%$ in untransformed Svevo plants (Table 1)

To evaluate the response to FCR, infection experiments with F. culmorum were performed on durum wheat seedlings (Zadoks stage 11) of Ubi-UGT transgenic and untransformed Svevo plants. Symptom progression was visually scored for a period of three weeks and was evaluated by using a disease
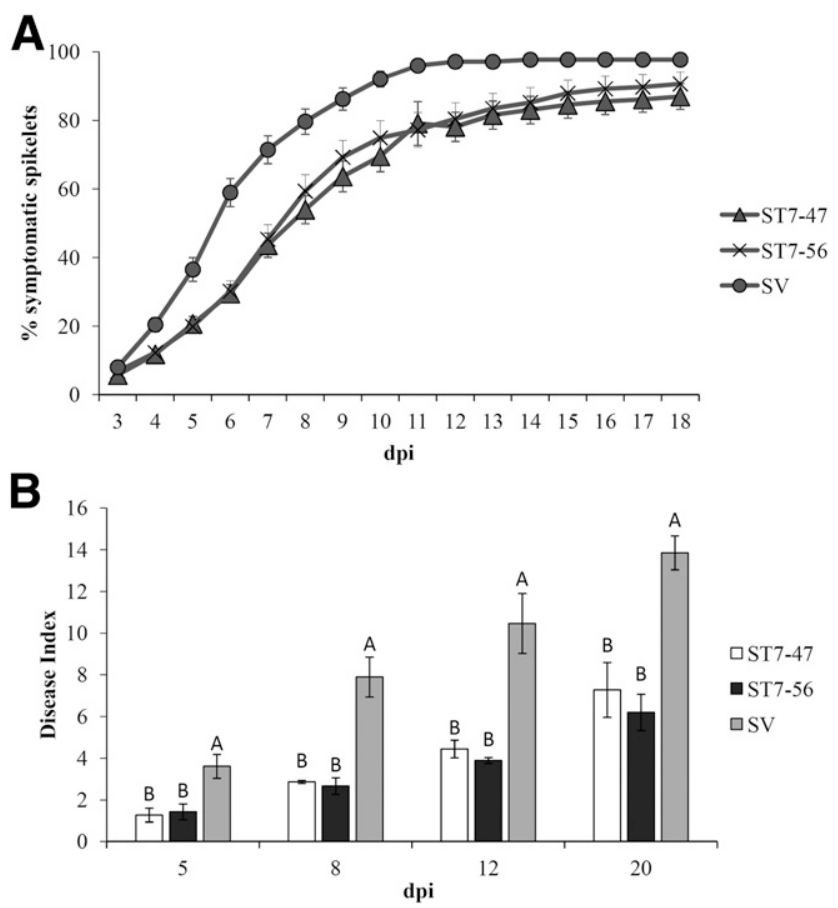

Fig. 4. Infection experiments on Triticum durum 'Svevo' (SV) and UbiUGT transgenic lines ST7-47 and ST7-56. A, Time-course development of Fusarium head blight (FHB) symptoms following Fusarium graminearum head inoculation. According to analysis of variance (ANOVA) and ranking of Tukey test, both Ubi-UGT lines were significantly different from Svevo from 5 to 9 days postinfection (dpi) $(P<0.01)$ and until $15 \mathrm{dpi}(P<0.05)$. B, Fusarium crown rot (FCR) symptom development following F. culmorum seedling inoculation. All data were subjected to ANOVA. Different letters correspond to significant differences $(P<0.01)$ according to the Tukey test. Data represent mean values \pm standard errors of three independent FHB and FCR assays. index (DI) that considers symptom extension and the browning index. Both transgenic lines exhibited less-severe symptoms compared with the untransformed plants, with a significant $50 \%$ reduction of the DI (Fig. 4B). Importantly, symptom reduction was maintained throughout the time of infection for both transgenic lines.

Finally, to assess the UGT-specific role, Ubi-UGT transgenic and untransformed Svevo plants were infected with the DONnonproducing fungal pathogen Bipolaris sorokiniana. No significant difference in response to leaf spot blotch disease was observed between transgenic and untransformed Svevo plants (Fig. 5), with the percentage symptomatic leaf area averaging 12 to $16 \%$ in both transgenic and wild-type leaves.

\section{Resistance to FHB increases}

\section{in transgenic Lem-UGT bread wheat.}

The Lem-UGT bread wheat lines, namely ST8-74 and ST849 , were also infected with $F$. graminearum to evaluate their response to FHB. In this case, a different trend of symptom progression was observed (Fig. 6). Disease severity was reduced by 20 to $40 \%$ in the ST8-74 line compared with Bobwhite untransformed plants, from 6 to 18 dpi (Fig. 6A). In line ST8-49 symptom severity was significantly reduced only between 6 and 12 dpi (Fig. 6B).

Harvested kernels from infected spikes of Lem-UGT lines, in particular of ST8-74, were more developed and filled than those of untransformed plants, with TKW of $8.1 \pm 0.2,6.4 \pm 0.4$, and $4.7 \pm 0.8$, respectively, in ST8-74, ST8-49, and Bobwhite plants.

UHPLC-mass spectrometry measurements of DON and D3G contents in flour extracted from mature kernels of Lem-UGT and untransformed plants revealed a higher DON+D3G content in both ST8-49 and ST8-74 lines as compared with Bobwhitederived flour (Table 2), though significantly only in line ST8-49 $(P<0.05)$. Despite this, the D3G content was 10 times greater in Lem-UGT lines than in untransformed plants. The D3G/DON ratio followed the same relative trend and, irrespective of the different UGT-expression levels among the two lines, it was 10 times greater, on average, in Lem-UGT lines compared with untransformed plants (Table 2).

\section{DISCUSSION}

Fusarium species, including $F$. graminearum and F. culmorum, cause devastating diseases for bread and durum wheat cultivation worldwide, namely FHB and FCR. Successful colonization of wheat heads by $F$. graminearum requires production of the trichothecene mycotoxins (Bai et al. 2001). Among them, DON is the most widely detected in diseased wheat and derived products (Canady et al. 2001; Streit et al. 2012). Among the natural mechanisms occurring in plants to reduce DON accumulation and, hence, to counteract its effects, DON conjugation to a glucose molecule by specific UGTs produces the less-toxic metabolite D3G; this is also considered a promising strategy for FHB resistance breeding (Kluger et al. 2015; Lemmens et al. 2005).

Table 1. Deoxynivalenol (DON) and deoxynivalenol-3-glucoside (D3G) content in wholemeal flour from seeds of infected spikes of Ubi-UGT (transgenic lines ST7-47 and ST7-56) and untransformed Svevo plants

\begin{tabular}{lccc}
\hline Genotype & DON (ppm) & D3G (ppm) & DON+D3G (ppm) \\
\hline Svevo & $454 \pm 1.1 \mathrm{~A}$ & $1.32 \pm 0.02 \mathrm{~B}$ & $455.3 \pm 4.5 \mathrm{~A}$ \\
ST7-56 & $0.68 \pm 0.01 \mathrm{~B}$ & $0.12 \pm 0.02 \mathrm{~B}$ & $0.80 \pm 0.1 \mathrm{~B}$ \\
ST7-47 & $5.36 \pm 0.05 \mathrm{~B}$ & $1.41 \pm 0.02 \mathrm{~A}$ & $0.16 \pm 0.03 \mathrm{~b}$ \\
\hline
\end{tabular}

${ }_{\mathrm{z}}$ Values are shown as parts per million (ppm) and represent means \pm standard errors detected in $1 \mathrm{mg}$ of flour. Different letters correspond to significant differences $(P<0.05$ in lower case, $P<0.01$ in upper case), according to the Tukey test. 
A major goal of this work was to evaluate the UGT-mediated response to FHB and FCR of transgenic durum wheat plants constitutively expressing the HvUGT13248 transgene (UbiUGT). Two Ubi-UGT lines challenged with $F$. graminearum at anthesis stage had appreciably reduced FHB severity (up to $30 \%)$ throughout the experiment, particularly significant $(P<$ 0.01 ) between 5 and $10 \mathrm{dpi}$, as compared with untransformed Svevo plants. Although seeds of all genotypes were clearly affected by the infection, DON content was significantly reduced in both transgenic lines. This is an important result, considering the toxic effects of DON on human and animal health (Maresca 2013; Pestka 2010). In Ubi-UGT plants, a more efficient DON detoxification from early infection stages may have impaired fungal progression and, as a consequence, decreased the amount of DON and D3G contamination in kernels. Such lines, in fact, had a much higher D3G/DON ratio (about 100 times) than Svevo untransformed plants, confirming their better DON-to-D3G conversion ability and the contribution of DON-detoxification in reducing FHB symptoms during disease progression (Li et al. 2015; Pasquet et al. 2016).

The extent of the UGT-based effect that we observed in durum wheat was lower than that of bread wheat lines expressing HvUGT13248, also point-inoculated with F. graminearum, reported by Li et al. (2015). However, results of the same authors from spray-inoculated plants in field trials, while confirming an overall significant reduction in FHB severity, were highly variable for DON, D3G, and DON+D3G contents, likely due to different disease pressures occurring through the years. A similar lack of correlation between disease severity and mycotoxin accumulation was also observed in various bread wheat genotypes (Mesterházy et al. 1999). Several reasons might account for the observed differences between our results on Ubi-UGT durum wheat plants and those of Li et al. (2015) on bread wheat. First, the tetraploid versus hexaploid genomic context could have impacted differently on the host-pathogen interaction and on DON-detoxification (Giancaspro et al. 2016). Furthermore, the use of different growth conditions (growth chamber versus greenhouse) and of $F$. graminearum strains with different aggressiveness (Akinsanmi et al. 2006) could also have influenced the experimental trend of the infection.

Seedlings of the Ubi-UGT lines were also challenged with $F$. culmorum, to evaluate the contribution of DON detoxification to FCR resistance. DON involvement as a virulence factor during durum wheat stem colonization but after initial

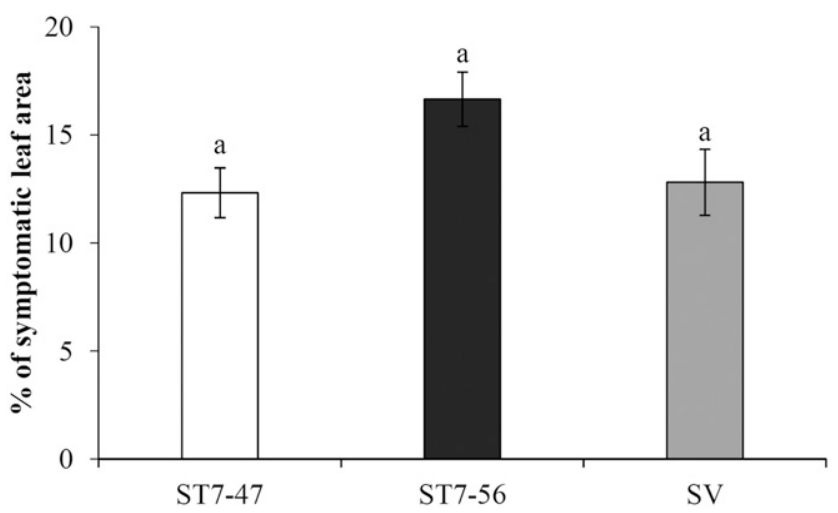

Fig. 5. Symptomatic leaf area following Bipolaris sorokiniana infection of Triticum durum 'Svevo' (SV) and Ubi-UGT transgenic lines ST7-47 and ST7-56. Data represent mean values \pm standard errors of three independent infection experiments. All data were subjected to analysis of variance. Different letters correspond to significant differences $(P<0.05)$ according to the Tukey test. infection was suggested by experiments with F. culmorum TRI6 mutants (Scherm et al. 2011). F. graminearum TRI5 mutants provided similar evidence when employed on bread wheat and soybean seedlings (Mudge et al. 2006; Sella et al. 2014) and so did a $F$. pseudograminearum TRI5 mutant on bread wheat (Powell et al. 2017). Our results indicated a symptom reduction of about $50 \%$ at all timepoints in the two Ubi-UGT durum wheat lines compared with Svevo wild-type seedlings. Notably, this is the first evidence of a direct involvement of DON detoxification by UGT glycosylation in the response to FCR. Using transcriptome analyses, Powell et al. (2017) identified a number of candidate genes differentially regulated after seedling inoculation with $F$. pseudograminearum, another causal agent of FCR. UGT and $A B C$-transporter genes were expressed only in infected samples, suggesting the involvement of detoxification. However, to date, none of these genes has been functionally characterized. Similarly, Wang et al. (2018) pointed out that TaUGT12887 and TaUGT3 wheat genes, known to be induced during FHB (Gottwald et al. 2012; Schweiger et al. 2013), were among those more rapidly and highly expressed in resistant seedlings following infection by F. graminearum.

In FCR, DON is produced even before visible symptoms can be observed (Mudge et al. 2006; Stephens et al. 2008) and its presence can impair root development (Masuda et al. 2007). The Ubi-UGT seedlings tested in the present work could have taken advantage of better root tolerance to DON, consequently displaying higher vigor than untransformed seedlings. Indeed, as observed by Stephens et al. (2008), fungal mycelium moves below the point of inoculation after germination and might, therefore, contribute to the presence of DON in the root environment under the soil surface. A severe impact on root development was highlighted in susceptible wheat genotypes as a
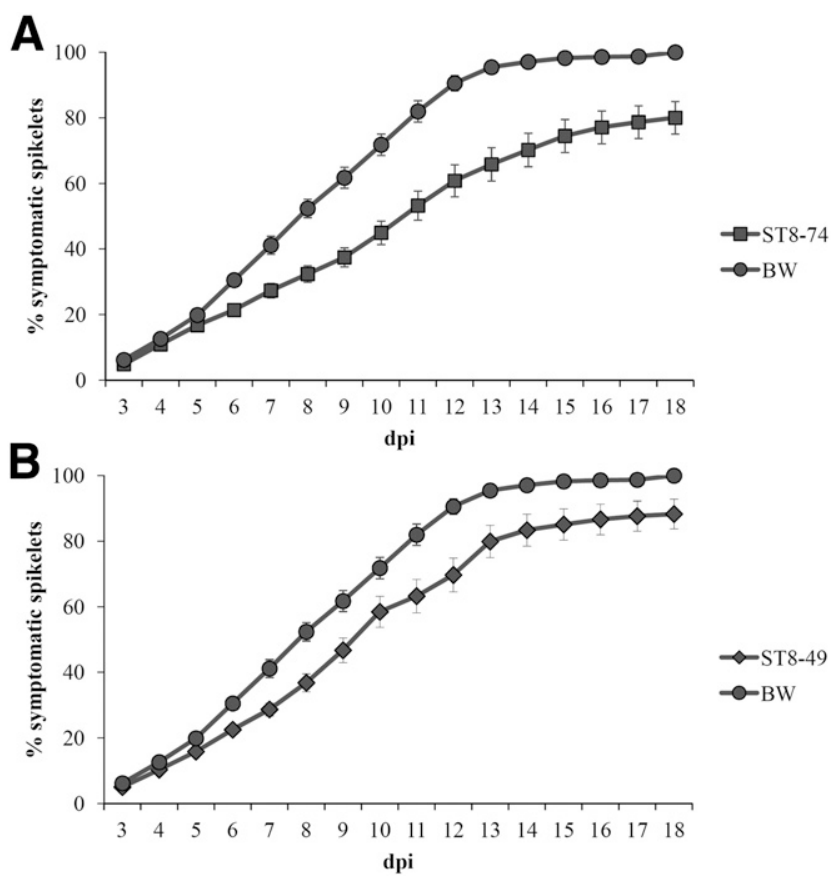

Fig. 6. Time-course of Fusarium head blight (FHB) symptom development following Fusarium graminearum inoculation of Triticum aestivum 'Bobwhite' (BW) and A, Lem-UGT transgenic lines ST8-74 and B, ST8-49. According to analysis of variance and ranking of Tukey test, the ST8-74 line was significantly different from Bobwhite between 6 and 12 days postinfection (dpi) $(P<0.01)$ and up to $18 \mathrm{dpi}(P<0.05)$, whereas for ST849 line the difference was between 6 and 12 dpi $(P<0.05)$. Data represent mean values \pm standard errors of three independent FHB assays. 
result of $F$. graminearum seedling infection (Wang et al. 2018). To our knowledge, none of the wheat genotypes tested in the natural population or in breeding programs exhibited both FHB and FCR resistance, as apparently conferred by the $H v U T G 13248$ transgene. As a remarkable exception, high FHB and FCR resistance was achieved by introgressing the $F h b-7 E L$ QTL from Thinopyrum elongatum (Ceoloni et al. 2017) and Fhb-7el 2 L from Thinopyrum ponticum (C. Ceoloni, unpublished data; Forte et al. 2014) into wheat. Improved FCR seedling resistance and FHB resistance was also achieved in wheat by cotransformation with Chi and Pep3 or Chi and MsrAl transgenes controlling different antifungal peptides (Liu et al. 2012). Although limited research has been carried out on simultaneous resistance to the two diseases, a genetic correlation between FHB and FCR resistance in bread wheat has not been found ( $\mathrm{Li}$ et al. 2010; Xie et al. 2006).

Our research indicates that the UGT-based effect on Fusarium diseases is specific to DON-producing pathogens, as the response of Ubi-UGT plants to $B$. sorokiniana infection was found not to differ from that of wild-type plants. Multiple resistance to different fungi, both hemibiotrophic or necrotrophic, like $F$. graminearum and $B$. sorokiniana, respectively, was described in transgenic wheat expressing different glycosidase inhibitors able to counteract fungal cell wall-degrading enzymes (Janni et al. 2008; Tundo et al. 2016b; Volpi et al. 2011). However, in that approach, host defense mechanisms commonly affected by different pathogens were involved, whereas UGTs specifically interact with mycotoxins. This aspect makes UGTs able to confer resistance against multiple Fusarium strains, as demonstrated by Li et al. $(2015,2017)$ with DON- or NIV-chemotypes of $F$. graminearum.

An additional target of this work was to test host reaction to FHB when the UGT-mediated DON-to-D3G conversion is active in the pathogen-targeted spike tissues. Our results from bread wheat plants expressing HvUGT13248 in spike-related tissues (Lem-UGT) pointed out their enhanced FHB resistance but, also, highlighted a differential contribution to resistance depending on the level of transgene expression. In fact, a doseeffect response was observed in the two transgenic lines compared with untransformed plants; the higher-expressing line (ST8-74) reduced FHB severity throughout the infection (nearly $40 \%$ maximum reduction), the lower-expressing line (ST8-49) reduced FHB severity to a lower extent (about 20 to $25 \%$ ) and, remarkably, at mid-infection stages. Li et al. (2015) observed up to $60 \%$ symptom reduction in the same bread wheat Bobwhite cultivar constitutively expressing $H v U G T 13248$. This difference is probably ascribable to lower disease pressure than in our experiments (70 versus $100 \%$, respectively). In both studies, transgenic UGT contribution to DON-to-D3G conversion significantly increased the D3G/DON ratio. We observed that the higher-expressing LemUGT line (ST8-74) reduced DON amount and enhanced D3G, indicating more efficient DON glycosylation due to higher UGT expression. By contrast, the lower-expressing ST8-49 line increased D3G content, compared with the control, while the DON content remained the same. The total DON (DON+D3G) was, therefore, even higher than in the control and in ST8-74.
This might have resulted from higher DON production and stronger aggressiveness of the pathogen in its interaction with this specific genotype.

The Leml promoter used to produce Lem-UGT lines, initially isolated from barley by Skadsen et al. (2002), drives gene expression in wheat lemma, palea, rachis, and anthers (Somleva and Blechl 2005; Tundo et al. 2016a). In wheat, the Leml-driven $P v P G I P 2$ transcript was highly expressed during head emergence, before anthesis (Tundo et al. 2016a), whereas the protein activity peaked at anthesis stage (Tundo et al. 2016a), similarly to the reporter green fluorescent protein (Somleva and Blechl 2005). A key factor in DON-sensitivity of the host plant seems to be the timing of toxin neutralization; the earlier the mycotoxin is neutralized, the higher are the chances of limiting pathogen spread (Li et al. 2015, 2017). Due to this, it is possible that Lemldriven expression of HvUGT13248 did not coincide with the time of maximum DON production in the fungus. Indeed, the HvUGT13248 protein might be degraded quickly after its transcriptional peak, hence being insufficient at later and more critical stages for effective plant response to DON. By contrast, under constitutive expression, like that of Ubil-driven $H v U G T 13248$, the transgene activity was likely to be relatively constant throughout all developmental stages. In addition, or as an alternative, the absence of ectopic UGT in ovaries and kernels could have contributed to the higher content of total DON+D3G in Lem-UGT derived flours than in control extracts. By contrast, DON+D3G amount was much lower in Ubi-UGT lines, indicating the importance of the 'promptness' of host detoxification to impair fungal DON production.

In conclusion, the present work provides evidence of the contribution of DON-detoxification in wheat resistance to Fusarium diseases. Based on the effect of the floral-specific expression on FHB symptoms, the need for a minimum UGT amount to effectively contribute to resistance is envisaged. The results concerning durum wheat indicate that both FHB and FCR diseases are positively affected by the UGT-based DON detoxification mechanism. This evidence is particularly noteworthy for a highly susceptible species like durum wheat. For both durum and bread wheat, the proved efficacy of DONdetoxifying UGT makes it an attractive trait in breeding programs targeting broad-spectrum resistance against Fusarium pathogens.

\section{MATERIALS AND METHODS}

Constructs preparation, production, and selection of wheat transgenic plants.

The pAHC17_Ubi1::HvUGT13248 and pAHC17_Lem1:: HvUGT13248 constructs were prepared by inserting the complete coding region of $H v U G T 13248$ into the BamHI site of pAHC17 (Christensen and Quail 1996) under control of the maize Ubiquitinl or the barley Leml promoters, with NOS terminator. The coding sequence of HvUGT13248 (accession number GU170355) was synthetized and inserted into pUC57 vector by GenScript Biotech (Piscataway, NJ, U.S.A.), with amino acidic sequence optimized for Triticum durum codon usage. A FLAG-tag region was added at the $3^{\prime}$ end of the

Table 2. Deoxynivalenol (DON) and deoxynivalenol-3-glucoside (D3G) content in wholemeal flour from seeds of infected spikes of Lem-UGT (transgenic lines ST8-49 and ST8-74) and untransformed Bobwhite plants ${ }^{\mathrm{Z}}$

\begin{tabular}{llrr}
\hline Genotype & DON (ppm) & D3G (ppm) & DON+D3G (ppm) \\
\hline Bobwhite & $412 \pm 20.2 \mathrm{a}$ & $36 \pm 20.7 \mathrm{~b}$ & $448 \pm 28.4 \mathrm{~b}$ \\
ST8-74 & $274 \pm 7.3 \mathrm{~b}$ & $313 \pm 36.8 \mathrm{a}$ & $587 \pm 29.4 \mathrm{ab}$ \\
ST8-49 & $414 \pm 6.8 \mathrm{a}$ & $320 \pm 66.3 \mathrm{a}$ & $1.14 \pm 0.15 \mathrm{a}$ \\
\hline
\end{tabular}

\footnotetext{
${ }^{\mathrm{z}}$ Values are shown as parts per million (ppm) and represent means \pm standard errors detected in 1 mg of flour. Different letters correspond to significant
} differences $(P<0.05)$, according to the Tukey test. 
$H v U G T 13248$. Two Bam $\mathrm{HI}$ restriction sites were added at the 5' and $3^{\prime}$ ends of the sequence, respectively. The HvUGT13248 sequence, as well as the pAHC17 and pAHC17_Lem1 (obtained from pAHC17_Lem1::PvPGIP2 [Tundo et al. 2016a] excising the PvPGIP2) were digested with BamHI (Promega, Milano, Italy) following the producer's protocol and were then ligated using the T4 ligase (Promega), generating pAHC17_Ubi1:: HvUGT13248 and pAHC17_Lem1::HvUGT13248 constructs (6,104 and 5,409 bp, respectively). The correct sequence and the insertion sites were confirmed by nucleic acid sequencing.

Constructs were introduced into immature embryo-derived calli of Svevo or Bobwhite, for durum and bread wheat, respectively, by biolistic bombardment, following the procedure described by Weeks et al. (1993). The constructs carrying the gene of interest (pAHC17_Ubi1::HvUGT13248 or pAHC17_Lem1::HvUGT13248) were cobombarded in a 3:1 molar ratio, with the pUbi::Bar construct carrying the Bar gene (accession number X17220.1), conferring resistance to phosphinotricin, used as selectable marker.

PCR was performed to discriminate the presence of transgenes in transformed plants. Genomic DNA was extracted from plant leaves using the method described by D'Ovidio and Porceddu (1996), obtaining a DNA solution of about $15 \mathrm{ng} / \mu \mathrm{l}$. PCR reactions were prepared in $10 \mu \mathrm{l}$ composed as follows: $5 \mu \mathrm{l}$ of GoTaq green master mix (Promega), $0.5 \mu \mathrm{M}$ of each specific primer, $1 \mu \mathrm{l}$ of extracted DNA, and nuclease-free sterile water for the remaining volume. Amplification conditions were 1 cycle at $95^{\circ} \mathrm{C}$ for $3 \mathrm{~min}$, then, 35 cycles of denaturation at $95^{\circ} \mathrm{C}$ for $45 \mathrm{~s}$, annealing at $60^{\circ} \mathrm{C}$ for $45 \mathrm{~s}$, extension at $72^{\circ} \mathrm{C}$ for $1 \mathrm{~min}$, and a final extension step at $72^{\circ} \mathrm{C}$ for $5 \mathrm{~min}$. Actin (accession number AB181991.1) was used as the control gene with a relatively consistent level of expression (housekeeping control). Amplicons were then confirmed by electrophoresis in $1 \%$ agarose gel.

To check the HvUGT13248 presence in Ubi-UGT or Lem-UGT plants, respectively, primer pairs used were Ubi-49F (TCGATGCT CACCCTGTTGTTT) and UGT472R (CTGCATGGTTGGGA GAAGAA) or Lem789F (ACCTTAACCTGGCGCCTTAG) and UGT472R, which produced amplicons of 522 and $788 \mathrm{bp}$, respectively. Ubi-49F and Bar2R (GAAACCCACGTCATGC CAGT) were used to check the presence of the Bar gene; TaAct77F (TCCTGTGTTGCTGACTGAGG) and TaAct321R (GGTCCAAACGAAGGATAGC) were employed to amplify the Actin gene.

\section{Characterization of durum wheat Ubi-UGT and bread wheat Lem-UGT transgenic lines.}

Southern hybridization was carried out as reported by Janni et al. (2008) on green leaf material. Hybridization probes labeled with digoxigenin were prepared by PCR following the procedure of D'Ovidio and Anderson (1994), using the primer pair UGT759F (AACCCTGCCATCGTACTACC) and FlagR (CTTGTCGTCGTCGTCCTTGTAGTC), amplifying almost $700 \mathrm{bp}$ of the HvUGT13248 coding region. Immunological detection with a chemiluminescence assay using 1:10,000 antidigoxigenin-AP' antibody (Roche Diagnostics, Monza, Italy) was performed by CSPD (Roche Diagnostics) methods, following the manufacturer's procedure.

For RT-PCR experiments, total RNA was extracted from leaf or spike (discarding ovaries) tissues, using Spectrum plant total RNA kit (Sigma-Aldrich, Milano, Italy), according to the manufacturer's instructions. Total RNA was qualitatively evaluated by electrophoresis in $1 \%$ agarose gel and was quantified in a Multiskan GO microplate spectrophotometer (Thermo Scientific, Monza, Italy). One microgram of total RNA was subjected to genomic DNA elimination, and first-strand cDNA synthesis was carried out, using a QuantiTect reverse transcription kit (Qiagen, Milano, Italy), according to the manufacturer's protocols.

RT-PCR reactions were prepared as described above, using $0.5 \mu \mathrm{l}$ of cDNA as template. Actin amplification was used as a housekeeping control. Amplicons were then confirmed by electrophoresis in $1 \%$ agarose gel. To check HvUGT13248 expression, the primer pair used was UGT35F (GCAC CACCTCATCGTCAGTC) and UGT1430R (GGATCCTCA CTTGTCGTCGTC), which produced an amplicon of 1,430 bp. TaAct77F and TaAct321R, which produce an amplicon of 350 and 250 bp on genomic (g)DNA and complementary (c) DNA, respectively, were used for Actin.

For Western blotting experiments, fresh or frozen $\left(-80^{\circ} \mathrm{C}\right)$ leaves or spikes of transgenic and untransformed plants were crushed in a mortar, with a pestle, using liquid nitrogen. Plant tissues were then homogenized with Laemmli buffer (Laemmli 1970), composed of $2 \%$ sodium dodecyl sulfate (SDS), $60 \mathrm{mM}$ Tris-HCl, $\mathrm{pH} 6.8,14.4 \mathrm{mM} \beta$-mercaptoethanol, and $10 \%$ glycerol $(2 \mathrm{ml} / \mathrm{g})$. Homogenized material was kept $15 \mathrm{~min}$ in ice and was then centrifuged at $12,000 \mathrm{rpm}$ for $20 \mathrm{~min}$ at $4^{\circ} \mathrm{C}$. The supernatant was recovered and the centrifugation step was repeated. Protein concentration of the total protein extracts was determined with the Bio-Rad Protein assay kit (Bio-Rad Laboratories, Segrate, Italy), using bovine serum albumin as standard. Ten micrograms of total proteins were separated in $15 \%$ polyacrylamide gel by SDS-polyacrylamide gel electrophoresis and were transferred to Immuno-Blot $0.2 \mu \mathrm{m}$ polyvinylidene diflouride membrane (Bio-Rad Laboratories). The polyclonal rabbit primary OctA-Probe antibody (1:2,000; Santa Cruz Biotechnology, Santa Cruz, CA, U.S.A.) coupled with horseradish peroxidase (HRP)-conjugated secondary antibody produced in rabbit $(1: 25,000$; Sigma-Aldrich) were used to detect the FLAG sequence presence at the N-terminus of HvUGT13248. Signals were detected, using Luminata Crescendo Western HRP substrate (Millipore, Bedford, MA, U.S.A.) as chemiluminescent substrate, by an autoradiography film.

\section{Root elongation assay.}

Sterilized wheat seeds of Ubi-UGT durum wheat lines and untransformed Svevo were sown in glass tubes containing Murashige Skoog (MS) rooting medium (MS salt mixture 2.15 $\mathrm{g} / \mathrm{liter}$, maltose $20 \mathrm{~g} / \mathrm{liter}$, thiamine- $\mathrm{HCl}$ [25 mg/500 ml] $5 \mathrm{ml} /$ liter, L-asparagine $0.075 \mathrm{~g} / \mathrm{liter}, 0.25 \% \mathrm{vol} / \mathrm{vol}$ phytagel) supplemented with $10 \mu \mathrm{M}$ DON (Romer Labs, Getzersdorf, Austria), diluted in $70 \%$ ethanol. Mock controls were prepared adding the same amount of $70 \%$ ethanol into the same medium without DON. Glass tubes were kept in the dark until germination (approximately 3 days after seeding) and were then moved to a growth chamber at $22^{\circ} \mathrm{C}$ with a 16 -h photoperiod $\left(300 \mu \mathrm{E} \mathrm{m}^{-2} \mathrm{~s}^{-1}\right)$. For each line, the experiment was performed in at least three replicates, with three to four seeds per replicate. Root growth was determined by measuring (in centimeters) the longest root of each seedling once a week for one month. The percentage of root inhibition was calculated as the difference between mock- and DON-treated samples for each line.

\section{Plant and fungal growth, infection assays, and DON/D3G analyses.}

Wheat seeds were surface-sterilized with sodium hypochlorite $(0.5 \%, \mathrm{vol} / \mathrm{vol})$ for $10 \mathrm{~min}$ and were then rinsed thoroughly in sterile water. After seed germination, seedlings were transferred into jiffy pots with soil and were vernalized at $4{ }^{\circ} \mathrm{C}$ for 2 weeks. Afterward, plants were, two by two, transferred in $14 \times 14 \mathrm{~cm}$ pots and were grown in a growth chamber at 18 to $23^{\circ} \mathrm{C}$ with a 14 -h photoperiod $\left(300 \mu \mathrm{E} \mathrm{m}^{-2} \mathrm{~s}^{-1}\right)$. The growth 
stages were assessed using the Zadoks' scale (Zadoks et al. 1974).

$F$. graminearum 3827 and $F$. culmorum UK99 were cultured on synthetic nutrient agar medium (Urban et al. 2002), to produce macroconidia. B. sorokiniana 62608 DSMZ strain was cultured on potato dextrose agar medium.

FHB infection experiments were performed in triplicate by single-spikelet inoculation with $F$. graminearum conidia, adjusted to $2.5 \times 10^{4}$ conidia/ml, using a final concentration of 500 conidia in $20 \mu \mathrm{l}$, supplemented with $0.05 \%$ Tween 20 . The conidia suspension $(20 \mu \mathrm{l})$ was pipetted directly through the glumes of two opposite central florets of a wheat head during anthesis (Zadoks stage 68). Infected spikes were covered with plastic bags for 2 days to maintain high humidity conditions. FHB disease symptoms were assessed, for a period of 18 days, by counting the number of visually diseased spikelets every day postinfection and by calculating their percentage out of the total number of spikelets of the respective head. For each experiment, at least 15 plants for each genotype were used. Bulked seeds obtained from infected plants of the respective genotype were weighed and TKW was calculated for each experiment.

FCR infection experiments were performed in triplicate, following the procedure described by Ceoloni et al. (2017). Seedlings were individually grown in jiffy pots and were arranged in plastic trays covered with a plastic film to maintain high relative humidity, at $22^{\circ} \mathrm{C}$ and with a $16 \mathrm{~h}$ light period. Inoculation was performed with a $F$. culmorum suspension adjusted to $1.5 \times 10^{6}$ conidia per milliliter, using a final concentration of $3 \times 10^{4}$ conidia in $20 \mu \mathrm{l}$, supplemented with $0.05 \%$ Tween 20. The inoculum was applied to the stem base leaf sheath at the first-leaf stage (Zadoks stage 11). Disease symptoms were assessed every three days, from day 5 to 21 postinoculation, using two parameters, symptom extension (SE, in centimeters) and browning index (BI) of the infected tissues (visual rating of the degree of extension of necrosis, as indicated by brown discoloration). The DI was subsequently determined as SE $\times$ BI (Beccari et al. 2011). For each experiment, at least 12 plants for each genotype were used.

The infection experiment to evaluate leaf spot blotch caused by $B$. sorokiniana was performed in triplicate on seedlings at the first leaf stage (Zadoks stage 11) by conidia inoculation of the upper surface of each leaf of transgenic and control wheat plants, as described by Janni et al. (2008). Disease symptoms were evaluated $72 \mathrm{hpi}$ by Assess image analysis software for plant disease quantification (American Phytopathological Society, St. Paul, MN, U.S.A.), as ratio between leaf area showing symptoms and the total leaf area. For each experiment, at least 10 plants for each genotype were used.

The levels of DON and D3G were determined in kernels of plants involving two $F$. graminearum infection experiments, using, in both cases, three technical replicates for each sample. DON and D3G metabolites were extracted from $100 \mathrm{mg}$ of wheat wholemeal flour in $400 \mu \mathrm{l}$ of $86: 14$ acetonitrile/water (vol/vol), by continuous shaking in a horizontal shaker for $24 \mathrm{~h}$ at $180 \mathrm{rpm}$ at $4^{\circ} \mathrm{C}$. The supernatant was recovered after centrifugation at $10,000 \times g$ for $10 \mathrm{~min}$. Supernatants $(20 \mu \mathrm{l})$ were injected into an UHPLC system (Ultimate 3000, Thermo Scientific) and were run in positive ion mode. The UHPLC system was coupled online with a mass spectrometer Q Exactive (Thermo Scientific), scanning in full mass spectrometry mode (two microscans) at 70,000 resolution in the 67 to $1,000 \mathrm{~m} / \mathrm{z}$ range. UHPLC-MS was performed as described by D'Alessandro et al. (2011). A Reprosil C18 column $(2.0 \times 150 \mathrm{~mm}, 2.5 \mu \mathrm{m})$ was used for metabolite separation. Data files were processed by MAVEN.52 (available online). Standard curves were obtained with serial dilutions of analytical standards of DON
(Romer Labs), resuspended in 96\% ethanol, and D3G (Romer Labs), $50 \mu \mathrm{g} / \mathrm{ml}$ in acetonitrile).

\section{Statistical analyses.}

All data were subjected to analysis of variance using SYSTAT12 software (Systat Software Inc., San Jose, CA, U.S.A.). The variable parameter (i.e., percentage of symptomatic spikelets for FHB, DI for FCR, quantification of DON/D3G content in flour) was considered as the dependent factor against the independent factors genotype and replicate. Two levels of significance $(P<0.05, P<0.01)$ were considered to assess significance of the $F$ values. When significant $F$ values were observed, a pairwise analysis was carried out by the Tukey honestly significant difference test at a 0.95 or 0.99 confidence level.

\section{ACKNOWLEDGMENTS}

The authors dedicate this work to the memory of Renato D'Ovidio, who conceived the project and coordinated the research. With great sorrow for us all, he passed away on March 2, 2017. We are thankful for the opportunity to be his students or colleagues. The authors acknowledge F. Favaron (University of Padova), S. A. Quarrie (Belgrade and Newcastle Universities), and P. J. Tricker (University of Adelaide) for critically reviewing the manuscript. Special thanks are due to C. Perani for technical assistance.

\section{LITERATURE CITED}

Akinsanmi, O. A., Backhouse, D., Simpfendorfer, S., and Chakraborty, S. 2006. Pathogenic variation of Fusarium isolates associated with head blight of wheat in Australia. J. Phytopathol. 154:513-521.

Bai, G. H., Desjardins, A. E., and Plattner, R. D. 2001. Deoxynivalenolnonproducing Fusarium graminerum causes initial infection but does not cause disease spread in wheat spikes. Mycopathologia 153:91-98.

Ban, T., Kishii, M., Ammar, K., Murakami, J., Lewis, J., William, M., Peña, R. J., Payne, T., Singh, R., and Trethowan, R. 2005. CIMMYT's challenges for global communication and germplasm enhancement for FHB resistance in durum and bread wheat. Pages 6-9 in: Proceedings of the 2005 National Fusarium Head Blight Forum; S. M. Canty, J. Lewis, L. Siler, and R. W. Ward, eds. Michigan State University, East Lansing, MI, U.S.A.

Ban, T., and Watanabe, N. 2001. The effects of chromosomes 3A and 3B on resistance to Fusarium head blight in tetraploid wheat. Hereditas 135: 95-99.

Beccari, G., Covarelli, L., and Nicholson, P. 2011. Infection processes and soft wheat response to root rot and crown rot caused by Fusarium culmorum. Plant Pathol. 60:671-684.

Berthiller, F., Dall'Asta, C., Schuhmacher, R., Lemmens, M., Adam, G. and Krska, R. 2005. Masked mycotoxins: Determination of a deoxynivalenol glucoside in artificially and naturally contaminated wheat by liquid chromatography-tandem mass spectrometry. J. Agric. Food Chem. 53:3421-3425.

Boenisch, M. J., and Schäfer, W. 2011. Fusarium graminearum forms mycotoxin producing infection structures on wheat. BMC Plant Biol. 11: 110.

Bowles, D., Lim, E.-K., Poppenberger, B., and Vaistij, F. E. 2006 Glycosyltransferases of lipophilic small molecules. Annu. Rev. Plant Biol. 57:567-597.

Buerstmayr, H., Ban, T., and Anderson, J. A. 2009. QTL mapping and marker-assisted selection for Fusarium head blight resistance in wheat: A review. Plant Breed. 128:1-26.

Canady, R., Coker, R., Rgan, S., Krska, R., Kuiper-Goodman, T., and Olsen, M. 2001. Safety evaluation of certain mycotoxins in food. WHO Food Additives Series: 47. Joint FAO/WHO Expert Committee on Food Additives. Rome.

Ceoloni, C., Forte, P., Kuzmanović, L., Tundo, S., Moscetti, I., De Vita, P., Virili, M. E., and D'Ovidio, R. 2017. Cytogenetic mapping of a major locus for resistance to Fusarium head blight and crown rot of wheat on Thinopyrum elongatum 7EL and its pyramiding with valuable genes from a Th. ponticum homoeologous arm onto bread wheat 7DL. Theor. Appl. Genet. 130:2005-2024.

Christensen, A. H., and Quail, P. H. 1996. Ubiquitin promoter-based vectors for high-level expression of selectable and/or screenable marker genes in monocotyledonous plants. Transgenic Res. 5:213-218. 
D’Alessandro, A., Gevi, F., and Zolla, L. 2011. A robust high resolution reversed-phase HPLC strategy to investigate various metabolic species in different biological models. Mol. Biosyst. 7:1024-1032.

D'Ovidio, R., and Anderson, O. D. 1994. PCR analysis to distinguish between alleles of a member of a multigene family correlated with wheat bread-making quality. Theor. Appl. Genet. 88:759-763.

D’Ovidio, R., and Porceddu, E. 1996. PCR-based assay for detecting IBgenes for low molecular weight glutenin subunits related to gluten quality properties in durum wheat. Plant Breed. 115:413-415.

Dellafiora, L., Dall'Asta, C., and Galaverna, G. 2018. Toxicodynamics of mycotoxins in the framework of food risk assessment-an in silico perspective. Toxins (Basel) 10:52.

Desmond, O. J., Manners, J. M., Stephens, A. E., Maclean, D. J., Schenk, P. M., Gardiner, D. M., Munn, A. L., and Kazan, K. 2008. The Fusarium mycotoxin deoxynivalenol elicits hydrogen peroxide production, programmed cell death and defence responses in wheat. Mol. Plant Pathol. 9:435-445.

Dyer, A. T., Johnston, R. H., Hogg, A. C., and Johnston, J. A. 2009. Comparison of pathogenicity of the Fusarium crown rot (FCR) complex (F. culmorum, F. pseudograminearum and F. graminearum) on hard red spring and durum wheat. Eur. J. Plant Pathol. 125:387-395.

Forte, P., Virili, M. E., Kuzmanović, L., Moscetti, I., Gennaro, A., D'Ovidio, R., and Ceoloni, C. 2014. A novel assembly of Thinopyrum ponticum genes into the durum wheat genome: Pyramiding Fusarium head blight resistance onto recombinant lines previously engineered for other beneficial traits from the same alien species. Mol. Breed. 34: 1701-1716.

Giancaspro, A., Giove, S. L., Zito, D., Blanco, A., and Gadaleta, A. 2016. Mapping QTLs for Fusarium head blight resistance in an interspecific wheat population. Front. Plant Sci. 7:1381.

Gottwald, S., Samans, B., Lück, S., and Friedt, W. 2012. Jasmonate and ethylene dependent defence gene expression and suppression of fungal virulence factors: Two essential mechanisms of Fusarium head blight resistance in wheat? BMC Genomics 13:369.

Ilgen, P., Hadeler, B., Maier, F. J., and Schäfer, W. 2009. Developing kernel and rachis node induce the trichothecene pathway of Fusarium graminearum during wheat head infection. Mol. Plant-Microbe Interact. 22:899-908

Janni, M., Sella, L., Favaron, F., Blechl, A. E., De Lorenzo, G., and D'Ovidio, R. 2008. The expression of a bean PGIP in transgenic wheat confers increased resistance to the fungal pathogen Bipolaris sorokiniana. Mol. Plant-Microbe Interact. 21:171-177.

Jansen, C., von Wettstein, D., Schäfer, W., Kogel, K.-H., Felk, A., and Maier, F. J. 2005. Infection patterns in barley and wheat spikes inoculated with wild-type and trichodiene synthase gene disrupted Fusarium graminearum. Proc. Natl. Acad. Sci. U.S.A. 102:1689216897.

Karlovsky, P. 2011. Biological detoxification of the mycotoxin deoxynivalenol and its use in genetically engineered crops and feed additives. Appl. Microbiol. Biotechnol. 91:491-504.

Kluger, B., Bueschl, C., Lemmens, M., Michlmayr, H., Malachova, A., Koutnik, A., Maloku, I., Berthiller, F., Adam, G., Krska, R., and Schuhmacher, R. 2015. Biotransformation of the mycotoxin deoxynivalenol in fusarium resistant and susceptible near isogenic wheat lines. PLoS One 10:e0119656.

Laemmli, U. K. 1970. Cleavage of structural proteins during the assembly of the head of bacteriophage T4. Nature 227:680-685.

Lemmens, M., Scholz, U., Berthiller, F., Dall'Asta, C., Koutnik, A., Schuhmacher, R., Adam, G., Buerstmayr, H., Mesterházy, A., Krska, R., and Ruckenbauer, P. 2005. The ability to detoxify the mycotoxin deoxynivalenol colocalizes with a major quantitative trait locus for Fusarium head blight resistance in wheat. Mol. Plant-Microbe Interact. 18:1318-1324.

Li, H. B., Xie, G. Q., Ma, J., Liu, G. R., Wen, S. M., Ban, T., Chakraborty, S., and Liu, C. J. 2010. Genetic relationships between resistances to Fusarium head blight and crown rot in bread wheat (Triticum aestivum L.). Theor. Appl. Genet. 121:941-950.

Li, X., Michlmayr, H., Schweiger, W., Malachova, A., Shin, S., Huang, Y., Dong, Y., Wiesenberger, G., McCormick, S., Lemmens, M., Fruhmann, P., Hametner, C., Berthiller, F., Adam, G., and Muehlbauer, G. J. 2017. A barley UDP-glucosyltransferase inactivates nivalenol and provides Fusarium head blight resistance in transgenic wheat. J. Exp. Bot. 68: 2187-2197.

Li, X., Shin, S., Heinen, S., Dill-Macky, R., Berthiller, F., Nersesian, N., Clemente, T., McCormick, S., and Muehlbauer, G. J. 2015. Transgenic wheat expressing a barley UDP-glucosyltransferase detoxifies deoxynivalenol and provides high levels of resistance to Fusarium graminearum. Mol. Plant-Microbe Interact. 28:1237-1246.
Liu, Z. W., Li, H. P., Cheng, W., Yang, P., Zhang, J. B., Gong, A. D., Feng, Y. N., Fernando, W. G. D., and Liao, Y. C. 2012. Enhanced overall resistance to Fusarium seedling blight and Fusarium head blight in transgenic wheat by co-expression of anti-fungal peptides. Eur. J. Plant Pathol. 134:721-732.

Lops, R., Pascale, M., Pancaldi, D., and Visconti, A. 1998. Infezioni fungine e presenza di deossinivalenolo in cariossidi di frumento prodotte in diverse regioni italiane. Informatore Fitopatologico 48:60-66.

Maier, F. J., Miedaner, T., Hadeler, B., Felk, A., Salomon, S., Lemmens, M., Kassner, H., and Schäfer, W. 2006. Involvement of trichothecenes in fusarioses of wheat, barley and maize evaluated by gene disruption of the trichodiene synthase (Tri5) gene in three field isolates of different chemotype and virulence. Mol. Plant Pathol. 7:449-461.

Maresca, M. 2013. From the gut to the brain: Journey and pathophysiological effects of the food-associated trichothecene mycotoxin deoxynivalenol. Toxins (Basel) 5:784-820.

Masuda, D., Ishida, M., Yamaguchi, K., Yamaguchi, I., Kimura, M., and Nishiuchi, T. 2007. Phytotoxic effects of trichothecenes on the growth and morphology of Arabidopsis thaliana. J. Exp. Bot. 58:1617-1626.

Mesterházy, Á., Bartók, T., Mirocha, C. G., and Komoróczy, R. 1999. Nature of wheat resistance to Fusarium head blight and the role of deoxynivalenol for breeding. Plant Breed. 118:97-110.

Miller, S. S., Chabot, D. M. P., Ouellet, T., Harris, L. J., and Fedak, G. 2004. Use of a Fusarium graminearum strain transformed with green fluorescent protein to study infection in wheat (Triticum aestivum). Can. J. Plant Pathol. 26:453-463.

Mudge, A. M., Dill-Macky, R., Dong, Y., Gardiner, D. M., White, R. G., and Manners, J. M. 2006. A role for the mycotoxin deoxynivalenol in stem colonisation during crown rot disease of wheat caused by Fusarium graminearum and Fusarium pseudograminearum. Physiol. Mol. Plant Pathol. 69:73-85.

Pasquet, J. C., Changenet, V., Macadré, C., Boex-Fontvieille, E., Soulhat, C., Bouchabké-Coussa, O., Dalmais, M., Atanasova-Pénichon, V., Bendahmane, A., Saindrenan, P., and Dufresne, M. 2016. A Brachypodium UDP-glycosytransferase confers root tolerance to deoxynivalenol and resistance to Fusarium infection. Plant Physiol. 172:559-574.

Payros, D., Alassane-Kpembi, I., Pierron, A., Loiseau, N., Pinton, P., and Oswald, I. P. 2016. Toxicology of deoxynivalenol and its acetylated and modified forms. Arch. Toxicol. 90:2931-2957.

Pestka, J. J. 2010. Deoxynivalenol: Mechanisms of action, human exposure, and toxicological relevance. Arch. Toxicol. 84:663-679.

Powell, J. J., Carere, J., Fitzgerald, T. L., Stiller, J., Covarelli, L., Xu, Q., Gubler, F., Colgrave, M. L., Gardiner, D. M., Manners, J. M., Henry, R. J., and Kazan, K. 2017. The Fusarium crown rot pathogen Fusarium pseudograminearum triggers a suite of transcriptional and metabolic changes in bread wheat (Triticum aestivum L.). Ann. Bot. 119:853-867.

Prat, N., Guilbert, C., Prah, U., Wachter, E., Steiner, B., Langin, T., Robert, O., and Buerstmayr, H. 2017. QTL mapping of Fusarium head blight resistance in three related durum wheat populations. Theor. Appl. Genet. 130:13-27.

Scherm, B., Balmas, V., Spanu, F., Pani, G., Delogu, G., Pasquali, M., and Migheli, Q. 2013. Fusarium culmorum: Causal agent of foot and root rot and head blight on wheat. Mol. Plant Pathol. 14:323-341.

Scherm, B., Orrù, M., Balmas, V., Spanu, F., Azara, E., Delogu, G. Hammond, T. M., Keller, N. P., and Migheli, Q. 2011. Altered trichothecene biosynthesis in TRI6-silenced transformants of Fusarium culmorum influences the severity of crown and foot rot on durum wheat seedlings. Mol. Plant Pathol. 12:759-771.

Schweiger, W., Boddu, J., Shin, S., Poppenberger, B., Berthiller, F., Lemmens, M., Muehlbauer, G. J., and Adam, G. 2010. Validation of a candidate deoxynivalenol-inactivating UDP-glucosyltransferase from barley by heterologous expression in yeast. Mol. Plant-Microbe Interact. 23:977-986.

Schweiger, W., Steiner, B., Ametz, C., Siegwart, G., Wiesenberger, G., Berthiller, F., Lemmens, M., Jia, H., Adam, G., Muehlbauer, G. J., Kreil, D. P., and Buerstmayr, H. 2013. Transcriptomic characterization of two major Fusarium resistance quantitative trait loci (QTLs), Fhb1 and Qfhs.ifa-5A, identifies novel candidate genes. Mol. Plant Pathol. 14: 772-785.

Sella, L., Gazzetti, K., Castiglioni, C., Schäfer, W., and Favaron, F. 2014. Fusarium graminearum possesses virulence factors common to Fusarium head blight of wheat and seedling rot of soybean but differing in their impact on disease severity. Phytopathology 104:1201-1207.

Shin, S., Torres-Acosta, J. A., Heinen, S. J., McCormick, S., Lemmens, M. Kovalsky Paris, M. P., Berthiller, F., Adam, G., and Muehlbauer, G. J. 2012. Transgenic Arabidopsis thaliana expressing a barley UDPglucosyltransferase exhibit resistance to the mycotoxin deoxynivalenol J. Exp. Bot. 63:4731-4740. 
Skadsen, R. W., Sathish, P., Federico, M. L., Abebe, T., Fu, J., and Kaeppler, H. F. 2002. Cloning of the promoter for a novel barley gene, Lem1, and its organ-specific promotion of GFP expression in lemma and palea. Plant Mol. Biol. 49:545-555.

Somleva, M. N., and Blechl, A. E. 2005. The barley Lem 1 gene promoter drives expression specifically in outer floret organs at anthesis in transgenic wheat. Cereal Res. Commun. 33:665-671.

Stack, R. W., Elias, E. M., Mitchell Fetch, J., Miller, J. D., and Joppa, L. R. 2002. Fusarium head blight reaction of Langdon durum-Triticum dicoccoides chromosome substitution lines. Crop Sci. 42:637-642.

Stephens, A. E., Gardiner, D. M., White, R. G., Munn, A. L., and Manners, J. M. 2008. Phases of infection and gene expression of Fusarium graminearum during crown rot disease of wheat. Mol. Plant-Microbe Interact. 21:1571-1581.

Streit, E., Schatzmayr, G., Tassis, P., Tzika, E., Marin, D., Taranu, I., Tabuc, C., Nicolau, A., Aprodu, I., Puel, O., and Oswald, I. P. 2012. Current situation of mycotoxin contamination and co-occurrence in animal feed-Focus on Europe. Toxins (Basel) 4:788-809.

Tundo, S. 2015. Exploitation of cell wall glycosidase inhibitors to improve wheat resistance against Fusarium graminearum. Ph.D. thesis. University della Tuscia, Aix-Marseille University, Italy.

Tundo, S., Janni, M., Moscetti, I., Mandalà, G., Savatin, D., Blechl, A., Favaron, F., and D'Ovidio, R. 2016a. PvPGIP2 accumulation in specific floral tissues but not in the endosperm limits Fusarium graminearum infection in wheat. Mol. Plant-Microbe Interact. 29:815-821.

Tundo, S., Kalunke, R., Janni, M., Volpi, C., Lionetti, V., Bellincampi, D., Favaron, F., and D'Ovidio, R. 2016b. Pyramiding PvPGIP2 and TAXI-III but not PvPGIP2 and PMEI enhances resistance against Fusarium graminearum. Mol. Plant-Microbe Interact. 29:629-639.

Ueno, Y., Nakajima, M., Sakai, K., Ishii, K., and Sato, N. 1973. Comparative toxicology of trichothec mycotoxins: Inhibition of protein synthesis in animal cells. J. Biochem. 74:285-296.
Urban, M., Daniels, S., Mott, E., and Hammond-Kosack, K. 2002. Arabidopsis is susceptible to the cereal ear blight fungal pathogens Fusarium graminearum and Fusarium culmorum. Plant J. 32: 961-973.

Volpi, C., Janni, M., Lionetti, V., Bellincampi, D., Favaron, F., and D'Ovidio, R. 2011. The ectopic expression of a pectin methyl esterase inhibitor increases pectin methyl esterification and limits fungal diseases in wheat. Mol. Plant-Microbe Interact. 24:1012-1019.

Wang, Q., Shao, B., Shaikh, F. I., Friedt, W., and Gottwald, S. 2018. Wheat resistances to Fusarium root rot and head blight are both associated with deoxynivalenol- and jasmonate-related gene expression. Phytopathology 108:602-616.

Weeks, J. T., Anderson, O. D., and Blechl, A. E. 1993. Rapid production of multiple independent lines of fertile transgenic wheat (Triticum aestivum). Plant Physiol. 102:1077-1084.

Wei, C.-M., Hansen, B. S., Vaughan, M. H., Jr., and McLaughlin, C. S. 1974. Mechanism of action of the mycotoxin trichodermin, a 12,13epoxytrichothecene. Proc. Natl. Acad. Sci. U.S.A. 71:713-717.

Xie, G., Zhang, M., Magner, T., Ban, T., Chakraborty, S., and Liu, C. 2006 Evidence that resistance to Fusarium head blight and crown rot are controlled by different genes in wheat. Pages 15-19 in: The global Fusarium initiative for international collaboration: a strategic planning workshop. T. Ban, J. M. Lewis, and E. E. Phipps, eds. CIMMYT, Batan, Mexico.

Zadoks, J. C., Chang, T. T., and Konzak, C. F. 1974. A decimal code for the growth stages of cereals. Weed Res. 14:415-421.

\section{AUTHOR-RECOMMENDED INTERNET RESOURCE}

MAVEN software: http://genomics-pubs.princeton.edu/mzroll 